\title{
The MicroRNA miR773 Is Involved in the Arabidopsis Immune Response to Fungal Pathogens
}

\author{
Raquel Salvador-Guirao,, ${ }^{1}$ Patricia Baldrich, ${ }^{1}$ Detlef Weigel, ${ }^{2}$ Ignacio Rubio-Somoza, ${ }^{1, \dagger}$ and \\ Blanca San Segundo ${ }^{1,3,+}$ \\ ${ }^{1}$ Centre for Research in Agricultural Genomics (CRAG) CSIC, IRTA, UAB, UB. Edifici CRAG. Carrer de la Vall Moronta. Campus \\ UAB, Bellaterra (Cerdanyola del Vallés), 08193 Barcelona, Spain; ${ }^{2}$ Department of Molecular Biology, Max Planck Institute for \\ Developmental Biology, 72076 Tübingen, Germany; and ${ }^{3}$ Consejo Superior de Investigaciones Científicas (CSIC), Barcelona, \\ Spain
}

Accepted 4 October 2017.

\begin{abstract}
MicroRNAs (miRNAs) are 21- to 24-nucleotide short noncoding RNAs that trigger gene silencing in eukaryotes. In plants, miRNAs play a crucial role in a wide range of developmental processes and adaptive responses to abiotic and biotic stresses. In this work, we investigated the role of miR773 in modulating resistance to infection by fungal pathogens in Arabidopsis thaliana. Interference with miR773 activity by target mimics (in MIM773 plants) and concomitant upregulation of the miR773 target gene METHYLTRANSFERASE 2 (MET2) increased resistance to infection by necrotrophic (Plectosphaerrella cucumerina) and hemibiotrophic (Fusarium oxysporum, Colletototrichum higginianum) fungal pathogens. By contrast, both MIR773 overexpression and MET2 silencing enhanced susceptibility to pathogen infection. Upon pathogen challenge, MIM773 plants accumulated higher levels of callose and reactive oxygen species than wild-type plants. Stronger induction of defense-gene expression was also observed in MIM773 plants in response to fungal infection. Expression analysis revealed an important reduction in miR773 accumulation in rosette leaves of plants upon elicitor perception and pathogen infection. Taken together, our results show not only that miR773 mediates pathogenassociated molecular pattern-triggered immunity but also demonstrate that suppression of miR773 activity is an effective approach to improve disease resistance in Arabidopsis plants.
\end{abstract}

Plants have evolved diverse mechanisms to defend themselves against pathogenic microorganisms. The initiation of defense responses typically begins with the recognition of conserved pathogen-associated molecular patterns (PAMPs) by host pattern-recognition receptors (PRR), which leads to PAMP-triggered immunity (PTI) (Jones and Dangl 2006; Boller and He 2009). During PTI, plants express a diverse array of immune responses, such as callose deposition and generation of reactive oxygen species (ROS), activation of $\mathrm{Ca}^{2+}$ signaling and protein phosphorylation cascades, which ultimately leads to the

Current address for Patricia Baldrich: Donald Danforth Plant Science Center. 975 North Warson Road, St. Louis, MO 63132, U.S.A.

${ }^{\dagger}$ Corresponding authors: Ignacio Rubio-Somoza;

E-mail: ignacio.rubio@cragenomica.es; and Blanca San Segundo; E-mail: blanca.sansegundo@cragenomica.es

*The $\boldsymbol{e}$-Xtra logo stands for "electronic extra" and indicates that six supplementary figures and two supplementary tables are published online.

(c) 2018 The American Phytopathological Society dynamic reprogramming of host gene expression and accumulation of pathogenesis-related (PR) proteins (Bigeard et al. 2015; van Loon et al. 2006). Among the PAMPs triggering defenses against pathogens are the bacterial (flagellin) and fungal (chitosan) elicitors (Luna et al. 2011; Zipfel et al. 2004). However, in order to survive, successful pathogens must produce and deliver effectors that effectively suppress PTI, resulting in disease susceptibility. As a counteractive strategy, plants have evolved a repertoire of immune receptors, or resistance (R) proteins, that recognize pathogen effectors to mount an immune response, the so-called effector-triggered immunity (ETI) (Cui et al. 2015; Jones and Dangl 2006). The plant hormones ethylene (ET), jasmonic acid (JA), and salicylic acid (SA) play central roles in the regulation of plant immune responses (De Vleesschauwer et al. 2014; Denancé et al. 2013; Glazebrook 2005; RobertSeilaniantz et al. 2011). While many of these responses rely on gene expression changes due to transcriptional activation or repression, there is increasing evidence for posttranscriptional regulation of immune responses, with host endogenous small RNAs being essential in both PTI and ETI (Huang et al. 2016; KatiyarAgarwal and Jin 2010; Pumplin and Voinnet 2013; Staiger et al. 2013; Seo et al. 2013).

MicroRNAs (miRNAs) are a class of short noncoding small RNAs that direct posttranscriptional gene silencing in a sequencespecific manner through cleavage or translational repression mechanisms (Axtell 2013; Brodersen et al. 2008; Llave et al. 2002; Rogers and Chen 2013). miRNAs inhibit the translation of target mRNAs on the endoplasmic reticulum in Arabidopsis (Li et al. 2013). MiRNAs play a critical role in controlling developmental processes ( $\mathrm{Li}$ and Zhang 2016; Rubio-Somoza and Weigel 2011) and adaptation to different types of abiotic (e.g., nutrient deficiency, drought, cold, and salinity) and biotic stresses (Campo et al. 2013; Chiou et al. 2006; Huang et al. 2016; Jagadeeswaran et al. 2009; Li et al. 2014; Navarro et al. 2006; Shivaprasad et al. 2012; Sunkar et al. 2012). Our knowledge of miRNA functions in plant immunity is, however, still limited.

The role of miRNAs in disease resistance was first described in the interaction of Arabidopsis thaliana with the bacterial pathogen Pseudomonas syringae (Navarro et al. 2006). Here, perception of flg22, a flagellin-derived peptide, promotes miR393 accumulation resulting in downregulation of auxin receptors and in bacterial resistance. Subsequently, several other miRNAs have been shown to play a role during defense in Arabidopsis, such as miR393, miR396, miR398b, miR4010, miR472, miR844, and miR863 (Boccara et al. 2014; Lee et al. 2015; Li et al. 2010; Navarro et al. 2006; Niu et al. 2016; Park et al. 2014; Soto-Suárez et al. 2017). miRNAs might function as 
positive or negative regulators in PTI and ETI by targeting negative or positive defense regulators. Although an important fraction of the Arabidopsis miRNAome is known to be regulated during pathogen infection (Baldrich et al. 2014; Jagadeeswaran et al. 2009), the function of most of these pathogen-regulated miRNAs and their contribution to Arabidopsis immunity remains elusive. Additionally, most studies so-far carried out for the identification of miRNAs affecting disease resistance defense have been executed in the interaction of Arabidopsis with the bacterial pathogen Pseudomonas syringae, and our knowledge of miRNAs controlling immune responses to infection by fungal pathogens in Arabidopsis is still scarce.
In the present work, we examined the role of miR773 in Arabidopsis immunity to fungal infection. We show that blocking miR773 activity through the expression of a miR773 target mimic in MIM773 lines and the concomitant upregulation of the miR773 target gene MET2 (Fahlgren et al. 2007; Li et al. 2010) confer resistance to infection by necrotrophic (Plectosphaerella cucumerina) and hemibiotrophic (Fusarium oxysporum f. sp. conglutinans, Colletotrichum higginsianum) fungal pathogens. Conversely, overexpression of MIR773A and silencing of $M E T 2$ increased susceptibility to fungal infection. Resistance to infection by $P$. cucumerina in MIM773 lines is associated with a stronger expression of basal defense responses upon pathogen attack, including higher accumulation
A

\section{P. cucumerina}
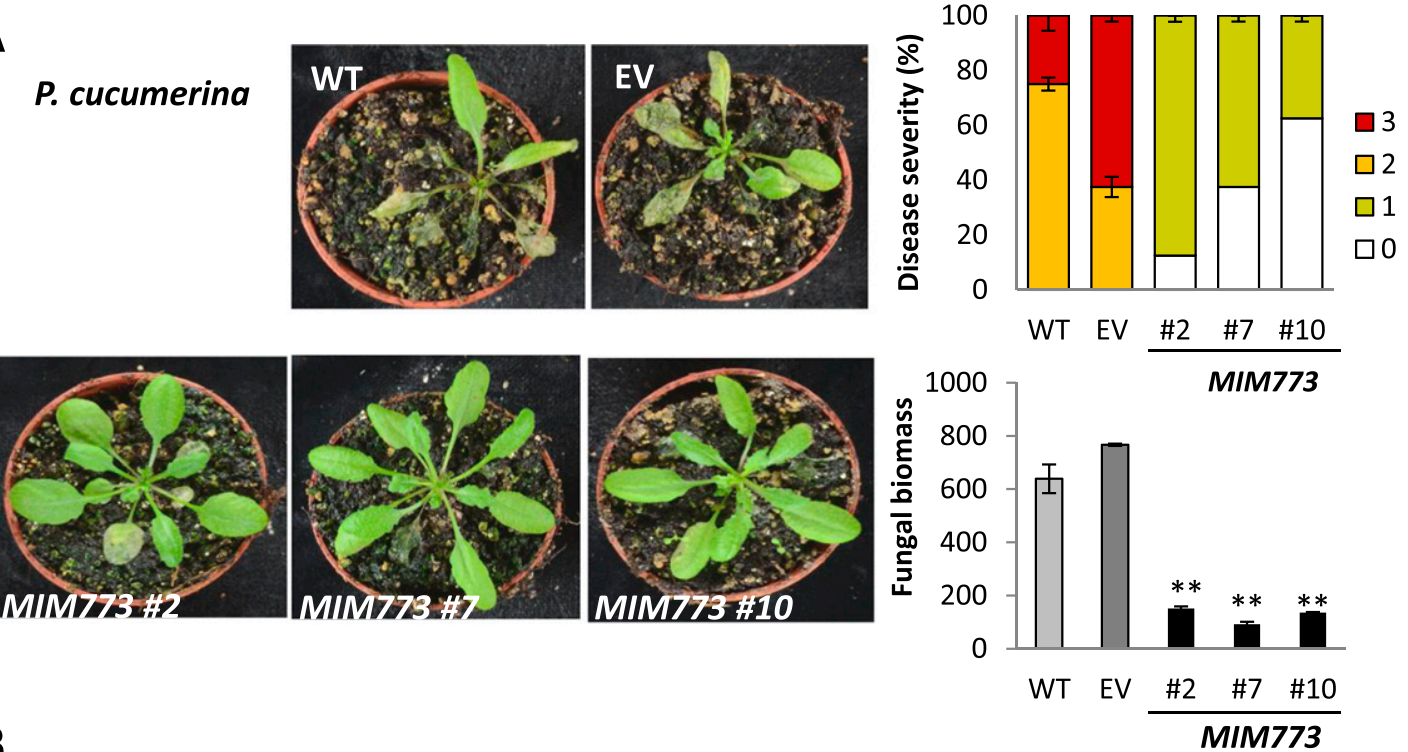

B
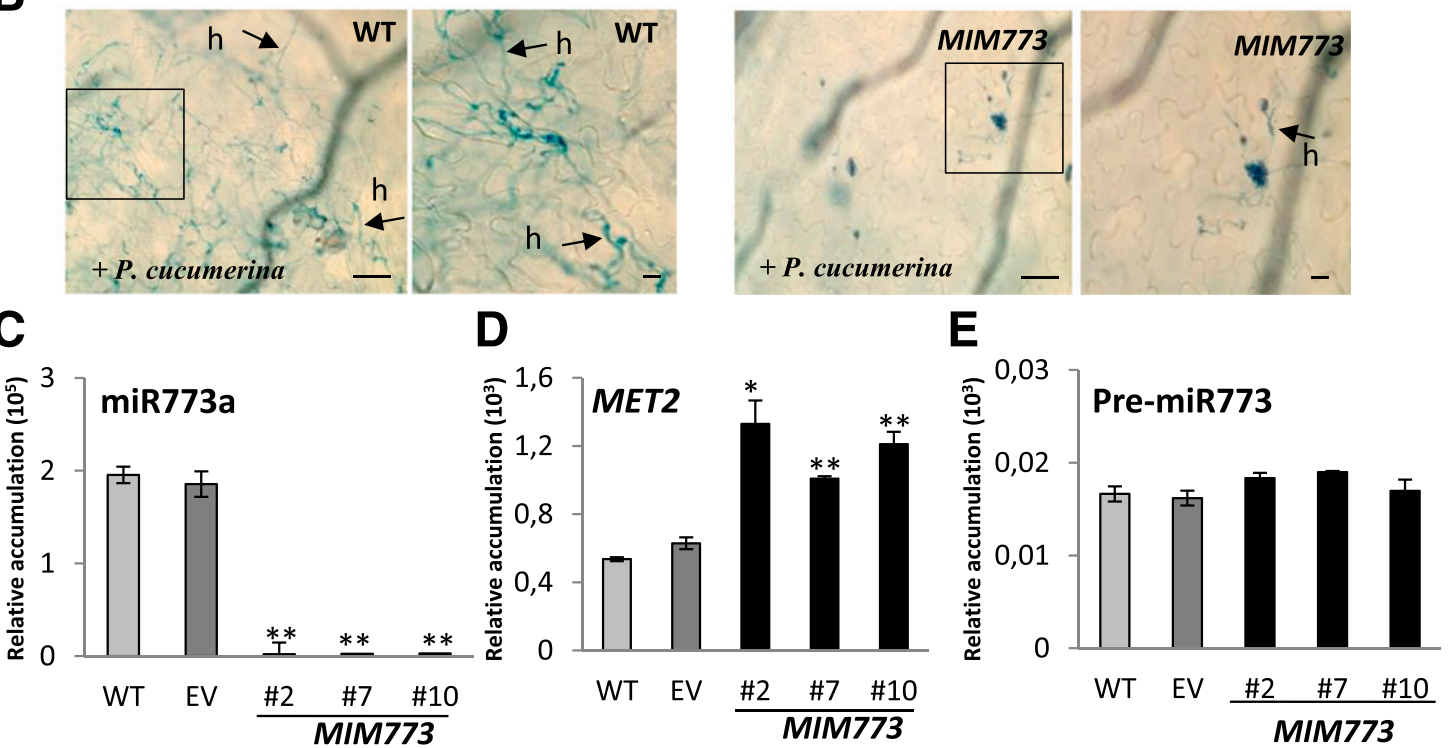

Fig. 1. MIM773 plants show resistance to infection by Plectosphaerrella cucumerina. Three-week old plants were inoculated with fungal spores. Results are from one of three independent experiments performed with three independent MIM773 lines (MIM773\#2, MIM773\#7, and MIM773\#10) and control plants (WT, empty vector) which gave similar results. At least 12 plants per genotype were assayed in each experiment. Histograms show the mean \pm standard deviation. Statistical significance (analysis of variance test, asterisks [**] indicate $P \leq 0.01$ ). A, Plants 10 days after spray-inoculation with $P$. cucumerina spores $\left(1 \times 10^{6}\right.$ spores per milliliter, $0.5 \mathrm{ml}$ per plant). Disease severity was scored as the number of leaves showing disease symptoms: $0=$ no symptoms, $1=1$ to $25 \%, 2=26$ to $50 \%, 3=51$ to $100 \%$. Quantification of fungal biomass was carried out by quantitative polymerase chain reaction (qPCR) using specific primers of P. cucumerina $\beta$-tubulin at 3 days postinoculation (Sánchez-Vallet et al. 2010). Values of fungal DNA were normalized to the Arabidopsis UBIQUITIN21 gene (At5g25760). B, Trypan blue staining of P. cucumerina-inoculated leaves at $24 \mathrm{~h}$ postinoculation. Right panels show the boxed region at higher magnification (scale bar, $200 \mu \mathrm{m}) ; \mathrm{h}=$ hyphae. C, Accumulation of mature miR773 was determined by stem-loop reverse transcription qPCR D, Accumulation of the miR773 target MET2, as determined by reverse transcription (RT)-qPCR. The single asterisk indicates a significance of $P<0.05$. E, Accumulation of miR773a precursor transcripts, as determined by RT-qPCR. 
of $\mathrm{H}_{2} \mathrm{O}_{2}$ and callose and superactivation of defense gene expression. Collectively, our results support a role of miR773 in controlling disease resistance in Arabidopsis.

\section{RESULTS}

Resistance to infection by fungal pathogens in MIM773 plants.

To identify miRNAs contributing to disease resistance in A. thaliana, we initially assayed resistance to infection by $P$. cucumerina in a set of miRNA target mimicry (MIM) lines in which the activity of miRNAs is suppressed and the activity of miRNA targets correspondingly increased (Todesco et al. 2010). P. cucumerina (previously known as Fusarium tabacinum; anamorph Plectosporium tabacinum) (Palm 1995) is a necrotrophic fungus that causes sudden death and blight in crops, such as melon, soybean, snap bean, pumpkin, squash, zucchini, or white lupine, and that can also infect Arabidopsis (Llorente et al. 2005; Sánchez-Vallet et al. 2012; Ton and MauchMani 2004).

Among the various MIM lines assayed (Supplementary Table S1), MIM773 plants were consistently more resistant to infection by $P$. cucumerina than wild-type and empty vector controls (Fig. 1A). Disease severity was defined as the number
A

F. oxysporum
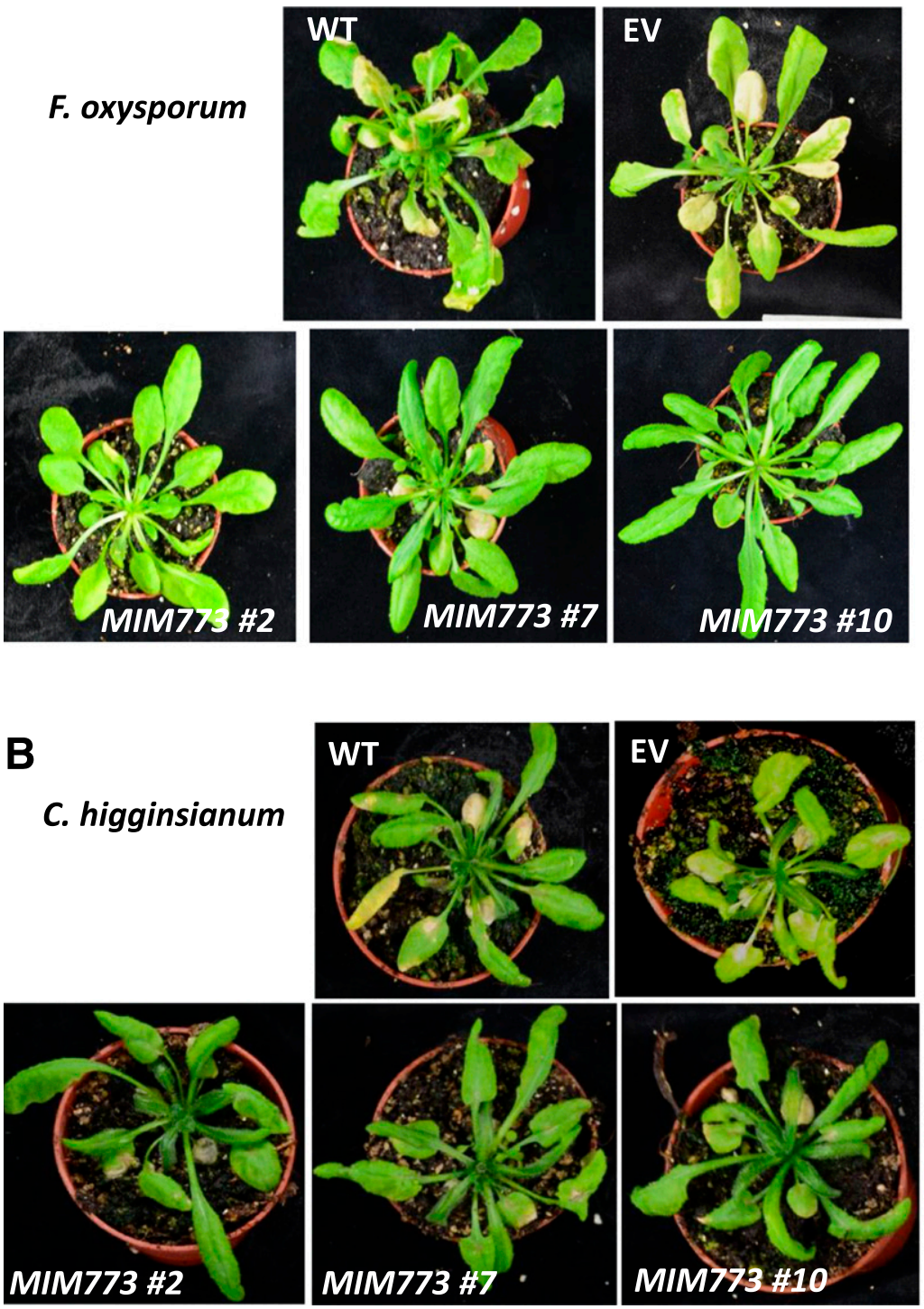
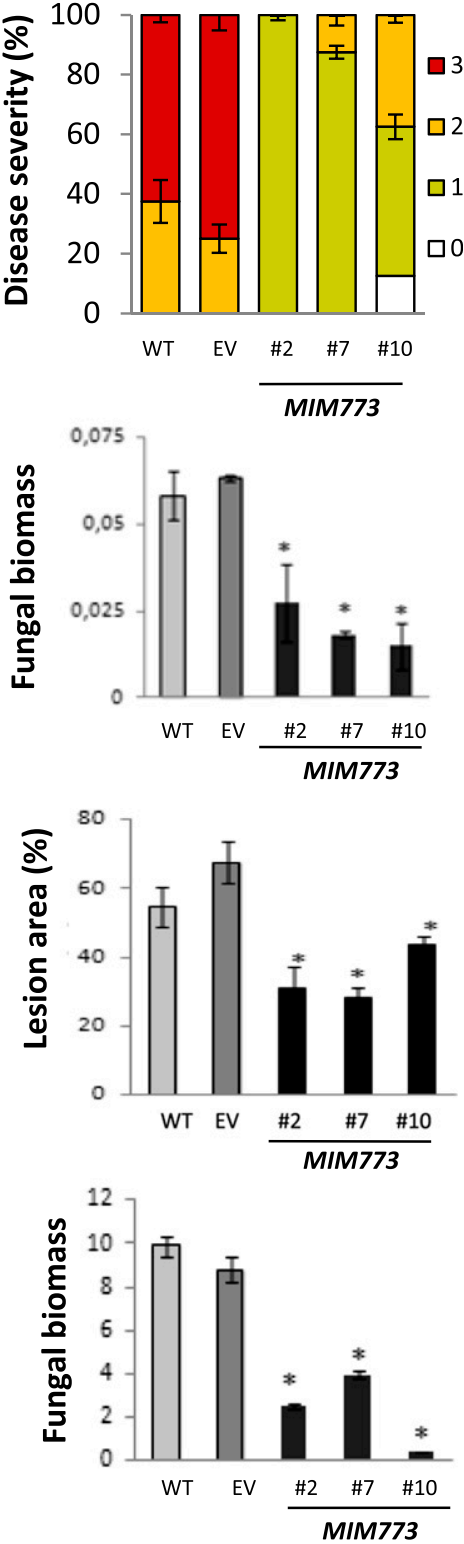

Fig. 2. MIM773 plants show resistance to infection by hemibiotroph fungal pathogens. Three-week-old plants were inoculated with fungal spores. Results are from one out of three independent experiments performed with three independent MIM773 lines (MIM773\#2, MIM773\#7, and MIM773\#10) and control plants (wild-type, empty vector), which gave similar results. At least 12 plants per genotype were assayed in each experiment. Histograms show the mean \pm standard deviation. Statistical significance (analysis of variance test, asterisks $\left[{ }^{*}\right]$ indicate $P \leq 0.05$ ). A, Plants 17 days after inoculation with Fusarium oxysporum $\mathrm{f}$. sp. conglutinans. Inoculation was carried out by applying the fungal spores $\left(1 \times 10^{6}\right.$ spores per milliliter) to soil. Disease severity was determined as described above. Quantification of F. oxysporum DNA was carried out by quantitative polymerase chain reaction (qPCR), using specific primers of the $F$. oxysporum chitinase class III gene at 3 days postinoculation (dpi) (Bravo Ruiz et al. 2016). Values are fungal DNA levels normalized against the Arabidopsis UBIQUITIN21 gene (At5g25760). B, Plants at 9 days after inoculation with Colletotrichum higginsianum. Leaves were locally inoculated with a spore suspension at $5 \times 10^{5}$ spores per milliliter. Diseased leaf area was quantified using image analysis software (Assess 2.0). Quantification of $C$. higginsianum DNA was carried out by qPCR using specific primers for the $C$. higginsianum ITS2 (Internally transcribed spacer 2) gene at 3 dpi. Values are fungal DNA levels normalized against the Arabidopsis UBIQUITIN21 gene. 
of plants falling into disease categories based on the number of diseased leaves. At 10 days after infection, the MIM773 plants had a disease score of 0 (plants with no symptoms) and 1 (plants with up to $25 \%$ of diseased leaves). By the same time, control plants scored in categories 2 (26 to $50 \%$ of diseased leaves) and 3 (51 to $100 \%$ ) (Fig. 1A). Measurements of fungal biomass by quantitative polymerase chain reaction (qPCR) (Fig. 1A) and trypan blue staining (Fig. 1B) confirmed reduced fungal growth in leaves of $P$. cucumerina-inoculated MIM773 plants compared with $P$. cucumerina-inoculated control plants.

To ascertain that the observed effects were due to changes in miR773 activity, we assayed miR773 accumulation and miR773 target gene expression. In Arabidopsis, there are two MIR773 family members, with miR773a having been shown to target MET2 (At4g14140) (Fahlgren et al. 2007; Li et al. 2010). As expected, MIM773 plants accumulated lower levels of mature miR773 as a consequence of mimicry-triggered miRNA degradation (Todesco et al. 2010) (Fig. 1C; Supplementary Fig. S1). As a control, miR396 accumulation was found not to be affected in MIM773 plants relative to control (wild-type and empty vector plants) (Supplementary Fig. S2). The observed reduction in mature miR773 levels was paralleled by an increase in MET2 transcript levels (Fig. 1D). MIR773A precursor transcripts accumulated at similar levels in MIM773 and wildtype plants (Fig. 1E). Finally, the MIM773 plants were similar to wild-type plants in terms of growth and rosette leaf number and diameter (Supplementary Fig. S3).

Next, we challenged MIM773 plants with the hemibiotrophic fungi Fusarium oxysporum f. sp. conglutinans and Colletotrichum higginsianum, the causal agents of wilt and of anthracnose leaf spot disease on a broad range of cultivated plant species as well as Arabidopsis (Mauch-Mani and Slusarenko 1994; O'Connell et al. 2004). Upon F. oxysporum f. sp. conglutinans inoculation, chlorosis appeared by 5 to 7 days postinoculation in leaves of control plants and progressively progressed over time, whereas by MIM773 lines exhibited much milder symptoms (Fig. 2A). Disease severity scores and fungal biomass supported a suppressive effect in MIM773 plants (Fig. 2A, right panels). MIM773 plants also were more resistant to $C$. higginsianum (Fig. 2B), with a smaller diseased leaf area and reduced fungal biomass accumulation (Fig. 2B, right panels).

Taken together, these results demonstrate that reduced miR773 activity enhances resistance to infection by both necrotrophic and hemibiotrophic fungi.

\section{MIM773 plants exhibit stronger PTI upon $P$. cucumerina infection.}

A common feature of basal resistance to pathogen infection is the accumulation of callose and ROS production at the infection sites (Luna et al. 2011; Morales et al. 2016; Pastor et al. 2013; Pétriacq et al. 2016; Torres et al. 2006). Callose functions as the first line of defense impeding fungal penetration, whereas ROS act as antimicrobial agents and signaling molecules for the activation of defense responses. Aniline blue staining indicated that MIM773 plants inoculated with $P$. cucumerina accumulated more callose than did wild-type plants and produced more $\mathrm{H}_{2} \mathrm{O}_{2}$ (Fig. 3A and B). It is noteworthy that, in MIM773 plants, callose and $\mathrm{H}_{2} \mathrm{O}_{2}$ were not detected before inoculation with
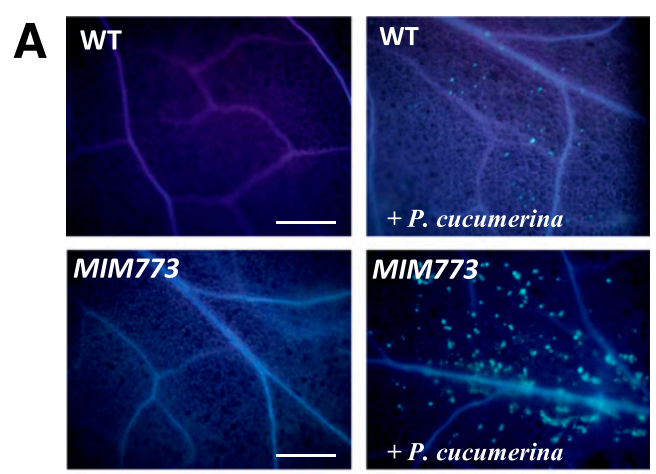

B
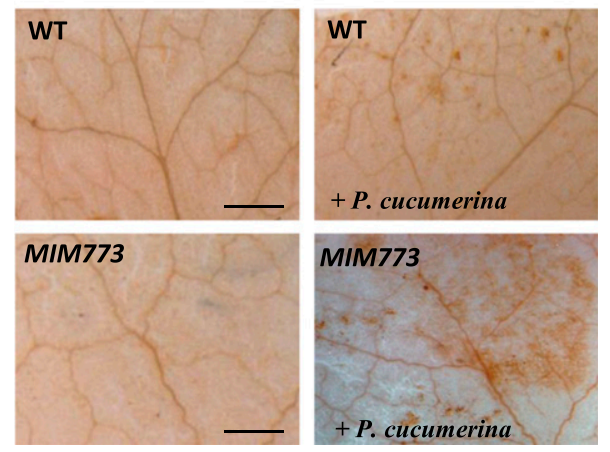
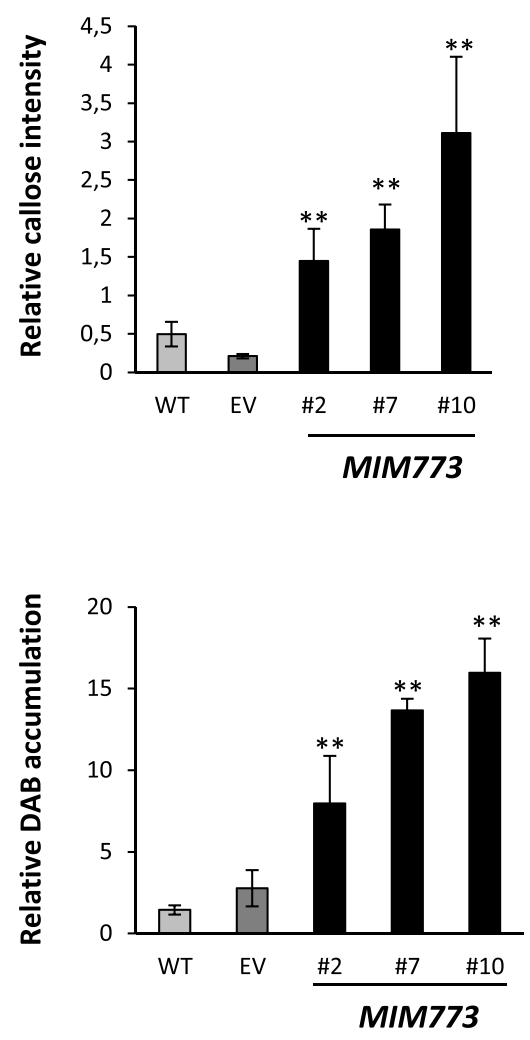

Fig. 3. Callose deposition and $\mathrm{H}_{2} \mathrm{O}_{2}$ production in Plectosphaerrella cucumerina-infected leaves of wild-type and MIM773 plants. In vitro-grown plants were sprayed with a $P$. cucumerina spore suspension $\left(1 \times 10^{6}\right.$ spores per milliliter, $2 \mathrm{ml}$ per plate). Statistical significance was determined by analysis of variance (asterisks [**] indicate $P \leq 0.01$ ). Representative results of one of three independent experiments that produced similar results are presented (three independent lines and 15 plants per independent line were assayed, and four leaves per plant were examined). Histograms (right panels) represent mean \pm standard deviation. A, Callose deposition in mock-inoculated (left panels) and P. cucumerina-inoculated (right panels) plants was examined by aniline blue staining at $24 \mathrm{~h}$ postinoculation (hpi). Callose deposition was quantified by determining the number of callose-corresponding pixels relative to the total number of pixels covering plant material on digital photographs. Bars (left panels) represent $50 \mu \mathrm{m} . \mathbf{B}, \mathrm{H}_{2} \mathrm{O}_{2}$ production was visualized by $3,3^{\prime}$-diaminobenzidine (DAB) staining at 24 hpi. Patches of brown precipitate of oxidized DAB are prominent in MIM773 plants. 
P. cucumerina, indicating that these immune responses were not constitutively active in MIM773 plants. The enhanced callose and $\mathrm{H}_{2} \mathrm{O}_{2}$ accumulation in leaves of MIM773 plants might well help to arrest pathogen growth in these plants.

\section{$M I R 773 A$ overexpression and $M E T 2$ silencing} confers enhanced susceptibility to $P$. cucumerina.

Since MIM773 plants exhibited enhanced resistance to fungal infection, we reasoned that either constitutive expression of MIR773A or silencing of MET2 expression might result in susceptibility to pathogen infection. We generated plants overexpressing MIR773A (MIR773A OE), which accumulated higher levels of mature miR773 and had reduced levels of MET2 transcripts (Fig. 4A). Northern blot analysis confirmed miR773 accumulation in MIR773 OE plants. In the absence of infection, MIR773A OE plants showed no obvious differences in growth, leaf number, or rosette diameter to nontransgenic plants (Supplementary Fig. S4).
Three-week-old MIR773A OE and control plants were challenged with $P$. cucumerina. MIR773A overexpression increased susceptibility to P. cucumerina (Fig. 4B). Disease susceptibility in $M I R 773 A$ OE plants was further confirmed by quantifying disease severity and fungal biomass in the infected plants (Fig. 4C). The opposite effects of depleting miR773 in MIM773 plants and overexpression of miR773 in MIR773A OE plants are in agreement with miR773 negatively regulating resistance to P. cucumerina.

We confirmed that, in two independent T-DNA insertion lines for MET2 (met2-1, SALK_010893 and met2-2, SALK_010896), the MET2 locus is disrupted by insertions in the second exon (Supplementary Fig. 5A). MET2 transcripts were not detected in the met 2 mutants, supporting that they are knockout mutants for MET2. As expected, MIR773A precursor expression was unaffected in the met 2 mutants, which showed no obvious morphological defects in the absence of infection.

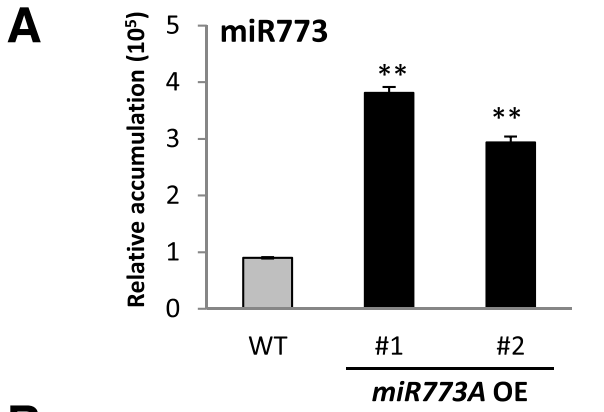

B
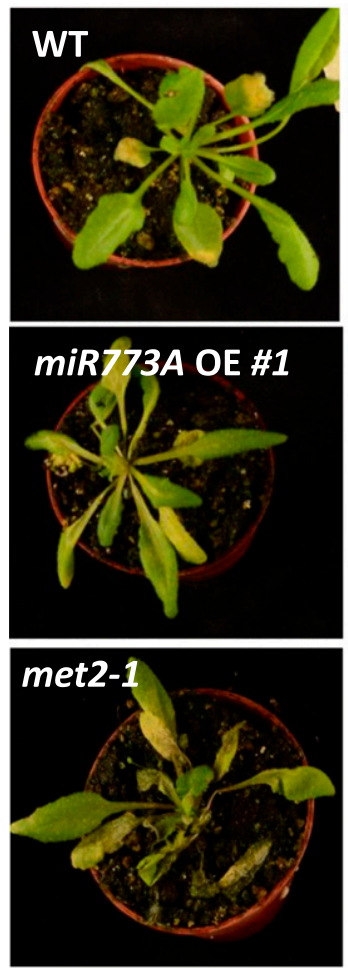
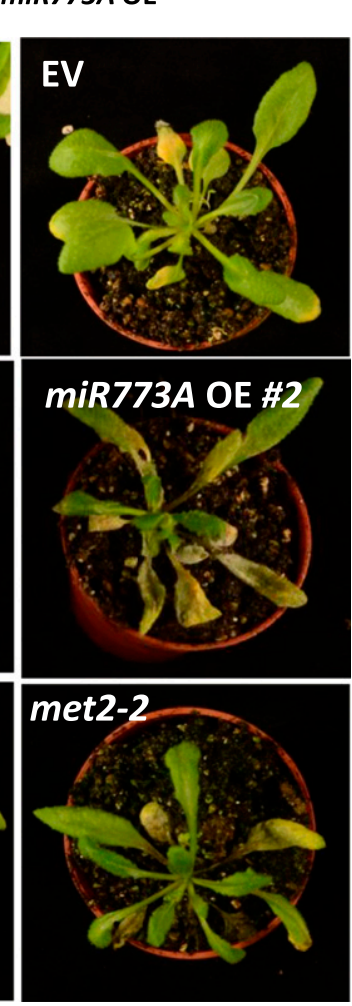

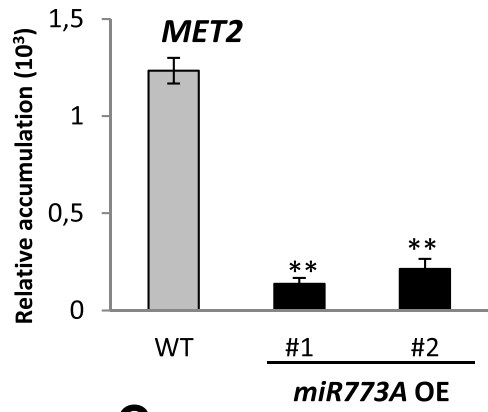

C
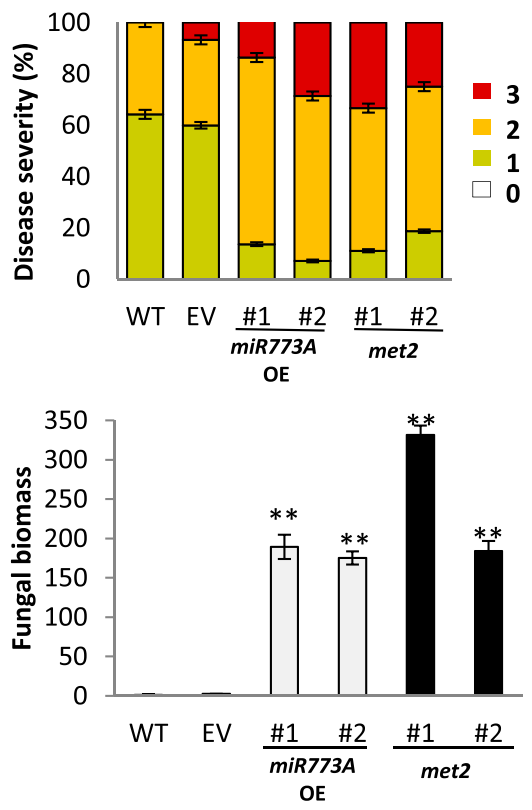

Fig. 4. Enhanced susceptibility to infection by Plectosphaerrella cucumerina in transgenic plants overexpressing (OE) MIR773A and met2 loss-of-function mutants. Three independent homozygous MIR773A OE plants, met2, and control plants (EV, empty vector; WT, wild-type) were analyzed. Three independent experiments were carried out, each with at least 12 plants per line. Results for two MIR773A OE lines are shown (similar results were obtained for the third MIR773A OE line). A, Accumulation of miR773 (left panel) and MET2 transcripts (right panel) in OE miR773a plants. The histogram shows the mean \pm standard deviation (analysis of variance test; asterisks [**] indicate $P \leq 0.01$ ). B, Disease susceptibility of OE miR773a and met 2 plants at 15 days postinoculation with $P$. cucumerina spores $\left(1 \times 10^{4}\right.$ spores per milliliter $)$. C, Disease severity was scored as the number of leaves showing disease symptoms: $0=$ no symptoms, $1=1$ to $25 \%, 2=26$ to $50 \%, 3=51$ to $100 \%$. Quantification of fungal biomass was carried out by quantitative polymerase chain reaction (qPCR) using specific primers of $P$. cucumerina $\beta$-tubulin at 3 days postinoculation (Sánchez-Vallet et al. 2010). Values of fungal DNA were normalized to the Arabidopsis UBIQUITIN21 gene (At5g25760). Statistical significance was determined by analysis of variance (asterisks $[* *]$ indicate $P \leq 0.01$, overexpressor or mutant lines vs. wild type). 
Upon challenge with $P$. cucumerina, both met 2 mutant lines exhibited enhanced susceptibility to infection, supported by visual inspection, disease severity scoring, and quantification of fungal biomass in the infected plants (Fig. 4B and C). This finding is consistent with the observed phenotype of susceptibility to $P$. cucumerina in MIR773A OE plants and provides additional support for the involvement of the miR773/MET2 pair in disease resistance.

\section{MiR773 accumulation decreases as part of PTI responses against fungal pathogens.}

Because altering miR773 activity has an effect on the host response to pathogen infection but does not appear to have consequences in the absence of pathogens, we sought to determine whether miR773 accumulation was itself pathogen-responsive.
As shown in Figure 5A, steady-state levels of MIR773 transcripts and mature miR773 rapidly decreased after infection with $P$. cucumerina, while expression of the miR773 target MET2 was increased (Fig. 5B).

In PTI, the induction of defense mechanisms relies on the detection of PAMPs (or elicitors). We therefore investigated whether miR773 might be induced by a crude preparation of fungal elicitors. Similar to $P$. cucumerina infection, elicitor treatment rapidly reduced both miR773 precursor and mature miRNA (Fig. 5C) and, at the same time, enhanced MET2 expression (Fig. 5D). These observations indicate that miR773 is PAMP-responsive and functions in PTI.

For a better understanding of miR773 regulation, we investigated miR773 accumulation in root and leaf tissues of wildtype plants in response to fungal infection. In unchallenged plants,
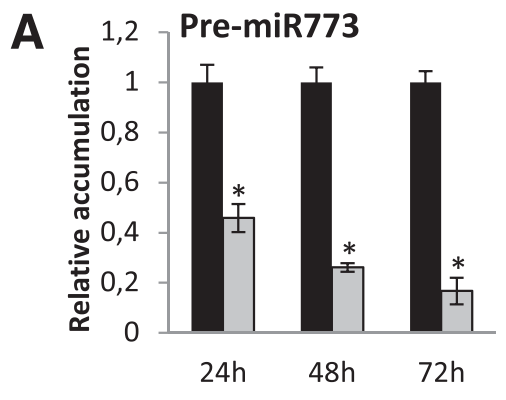

B
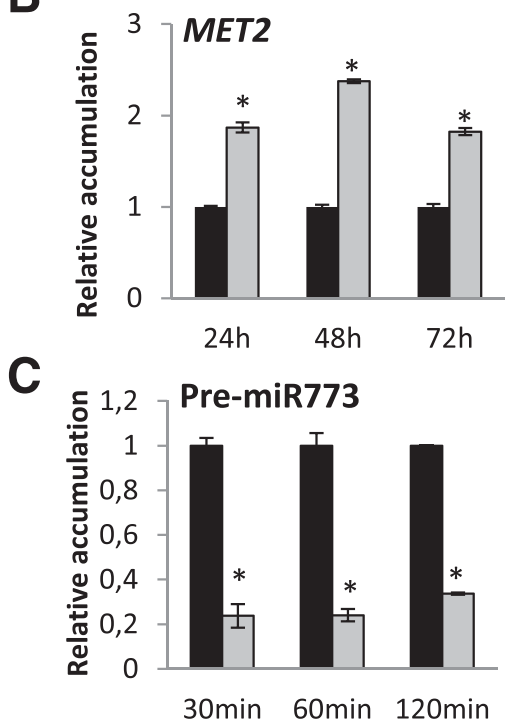

D

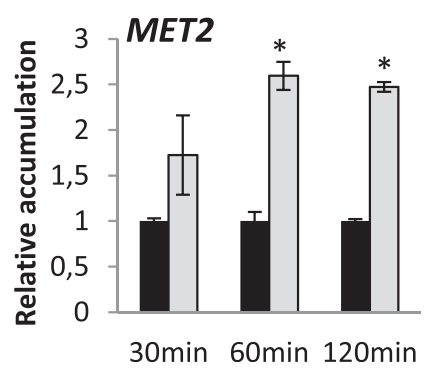

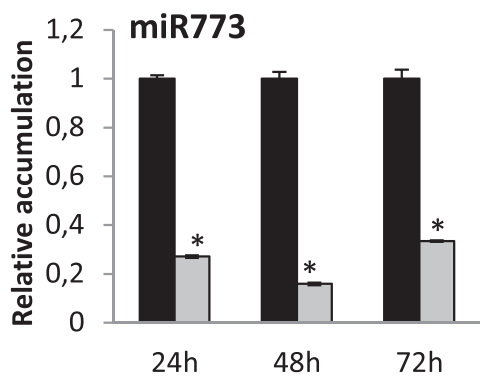

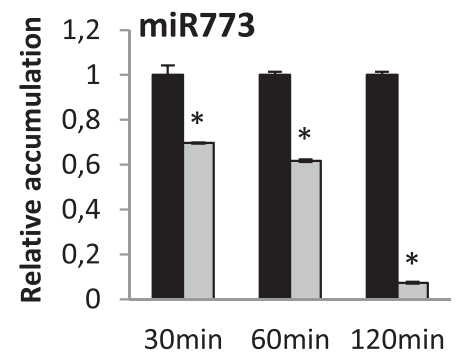

Fig. 5. Accumulation of miR773 and MET2 transcripts in rosette leaves in response to infection by Plectosphaerrella cucumerina or treatment with elicitors obtained from $P$. cucumerina. The values represent changes in the accumulation of miR773 sequences at the indicated times after inoculation with fungal spores, treatment with fungal elicitors, or mock-inoculation. Black and gray bars correspond to mock-inoculated and $P$. cucumerina-inoculated plants, respectively. The expression level in mock-inoculated plants was set to 1.0. Histograms show the mean \pm standard deviation of one of three biological replicates with similar results, each replicate with 12 plants per genotype (asterisks [*] indicate $P \leq 0.05$; analysis of variance test). A, Accumulation of premiR773 and mature miR773 sequences after inoculation with $P$. cucumerina spores, as determined by reverse transcription-quantitative polymerase chain reaction (RT-qPCR) and stem-loop RT-qPCR analysis, respectively. B, Accumulation of MET2 in response to inoculation with $P$. cucumerina spores. C, Accumulation of premiR773 and mature miR773 sequences at the indicated times of treatment with $P$. cucumerina elicitors. D, Accumulation of $M E T 2$ in response to treatment with $P$. cucumerina elicitors. 
miR773 expression was much higher in leaves than in roots (Fig. 6). Infection with either P. cucumerina or C. higginsianum significantly decreased miR773 accumulation in leaves but not in roots of wild-type plants (Fig. 6A, left and middle panels). In contrast, infection with the root pathogen $F$. oxysporum f. sp. conglutinans causes a reduction in miR773 levels in both tissues (leaves and roots) (Fig. 6A, right panel). The in-situ hybridization revealed miR773 accumulation in leaf mesophyll cells of noninfected plants (Fig. 6B, upper left panel), while miR773 was barely detectable in leaves of either uninfected and P. cucumerina-infected MIM773 plants or infected wild-type plants (Fig. 6B, upper panels). Collectively, these results demonstrate that miR773 accumulation in mesophyll cells of rosette leaves decreases in response to infection by $P$. cucumerina.

\section{miR773 reduction leads to stronger induction} of defense gene expression.

To ascertain the molecular basis of the enhanced resistance to P. cucumerina in MIM773 plants, we studied the expression of defense marker genes associated with JA/ET (PDF1.2), JA (VSP2), and SA (PR1,NPR1) signaling. Defense gene expression was induced in response to fungal infection in wild-type plants (Fig. 7A and B). Most importantly, transcripts of all defense genes accumulated to higher levels in $P$. cucumerina-inoculated MIM773 plants than in $P$. cucumerina-inoculated wild-type plants
(Fig. 7A and B). Contrary to this, a lower induction (VSP2, PRIa) or no induction $(P D F 1.2)$ of defense gene expression was observed in fungal-infected met 2 plants compared with the fungalinfected wild-type plants (Supplementary Fig. S6). These observations support the idea that, upon challenge with $P$. cucumerina, the MIM773 plants show stronger activation of defense responses that are regulated by ET, JA, or SA, consistent with the enhanced disease resistance in these plants. In addition, under non-infection conditions, the expression of PDF1.2 was slightly higher in MIM773 plants (Fig. 7A).

When examining the expression of genes involved in ET biosynthesis in MIM773 plants, namely ACS1 (1AMINOCYCLOPROPANE-1-CARBOXYLIC ACID SYNTHASE 1) and $A C O 2$ (ACC OXIDASE 2), they were found to be induced more strongly in MIM773 plants than in wild-type plants, in response to $P$. cucumerina infection (Fig. 7C). In the absence of pathogen infection, $A C S 1$ and $A C O 2$ expression was already slightly elevated in MIM773 plants (Fig. 7C).

These pieces of evidence support that MIM773 plants respond more strongly to pathogen infection through the superinduction of defense genes whose expression is regulated by defense-related hormones. An opposite response occurs in met 2 plants, these plants exhibiting a weaker induction (even no induction) of defense gene expression in response to fungal infection.

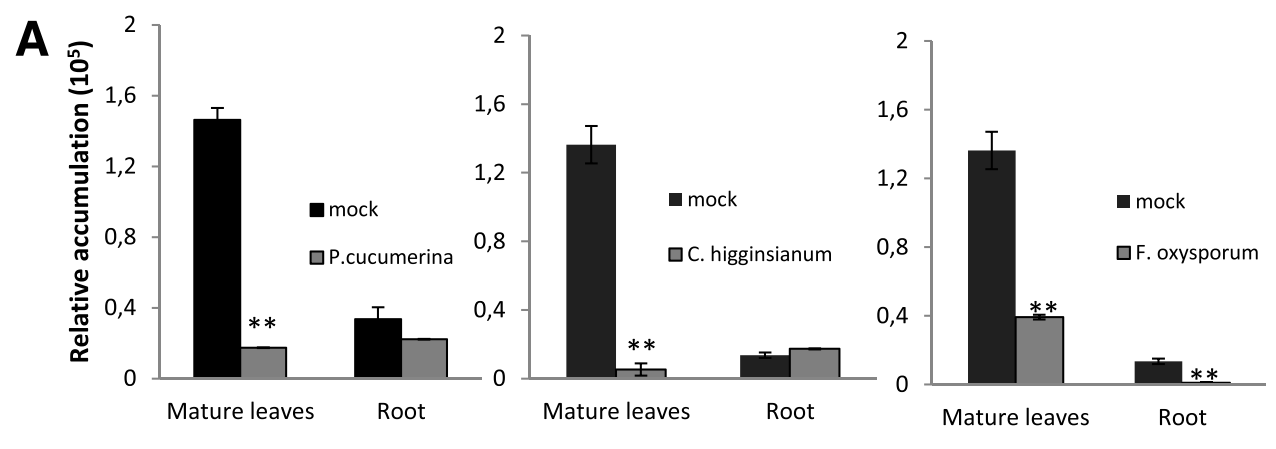

B
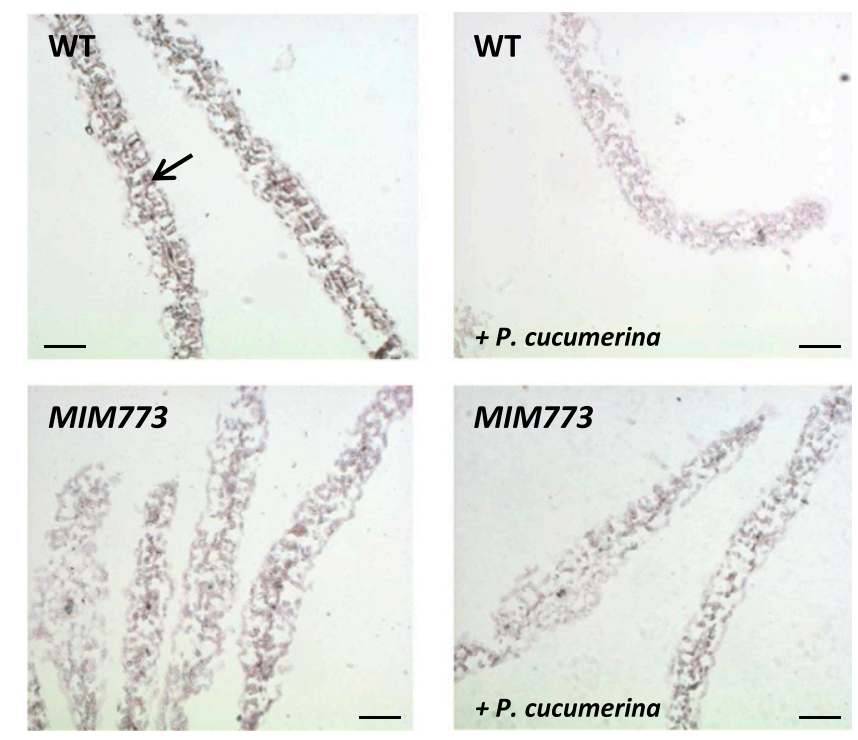

Fig. 6. Detection of miR773 in wild-type and MIM773 plants. A, Accumulation of miR773 was determined by stem-loop reverse transcription-quantitative polymerase chain reaction analysis in leaves and roots of in vitro-grown Arabidopsis plants at 24 h postinoculation with fungal spores (Plectosphaerrella cucumerina, $1 \times 10^{6}$ spores per milliliter; Colletotrichum higginsianum, $5 \times 10^{5}$ spores per milliliter; Fusarium oxysporum $\mathrm{f}$. sp. conglutinans, $1 \times 10^{6}$ spores per milliliter) (gray bars). Control plants were mock-inoculated (black bars). Histograms represent the mean \pm standard deviation of one of three biological replicates with similar results, each replicate with 24 plants per genotype. Statistical significance was determined by analysis of variance (asterisks (**) indicate $P \leq 0.01$, infected vs. mock-inoculated). B, In situ miR773 hybridization in mature leaves of $P$. cucumerina- and mock-inoculated plants. Hybridization was carried out using a $3^{\prime}$ digoxygenin-labeled locked nucleic acid miR773a probe (scale bar, $500 \mu \mathrm{m}$ ). 


\section{DISCUSSION}

In this study, we provide evidence that miR773a functions as a negative regulator of Arabidopsis immunity against necrotrophic (P. cucumerina) and hemibiotrophic ( $F$. oxysporum f. sp. conglutinans, $C$. higginsianum) fungal pathogens. Our results with MIM773 plants, in which miR773 activity is attenuated, and with plants deficient for the miR773 target MET2 extends previous findings that flagellin treatment results in downregulation of miR773 and that MIR773A overexpression increases susceptibility to infection by the hemibiotrophic bacterial pathogen P. syringae (Li et al. 2010).

MIM773 plants apparently mount ET/JA- and SA- dependent defense responses. Arabidopsis plants are known to deploy
JA/ET-, SA-, abscisic acid, and auxin-dependent defenses against $P$. cucumerina (García-Andrade et al. 2011; Llorente et al. 2008; Pétriacq et al. 2016; Sánchez-Vallet et al. 2012). Pathogen-induced downregulation of miR773 and upregulation of its target MET2 may, thus, lead to a potentiated activation of defense responses. Although we provide evidence for a function of miR773 in the regulation of ET/JA- and SA-dependent defense responses, our current understanding does not allow us to determine how miR773 and its target gene MET2 modulate the hormone-dependent activation of defense responses.

Given that MET2 is targeted by miR773, the question arises as to what role miR773-guided regulation of MET2 expression has in immunity. DNA methyltransferases and DNA demethylases function in an antagonistic manner in controlling the
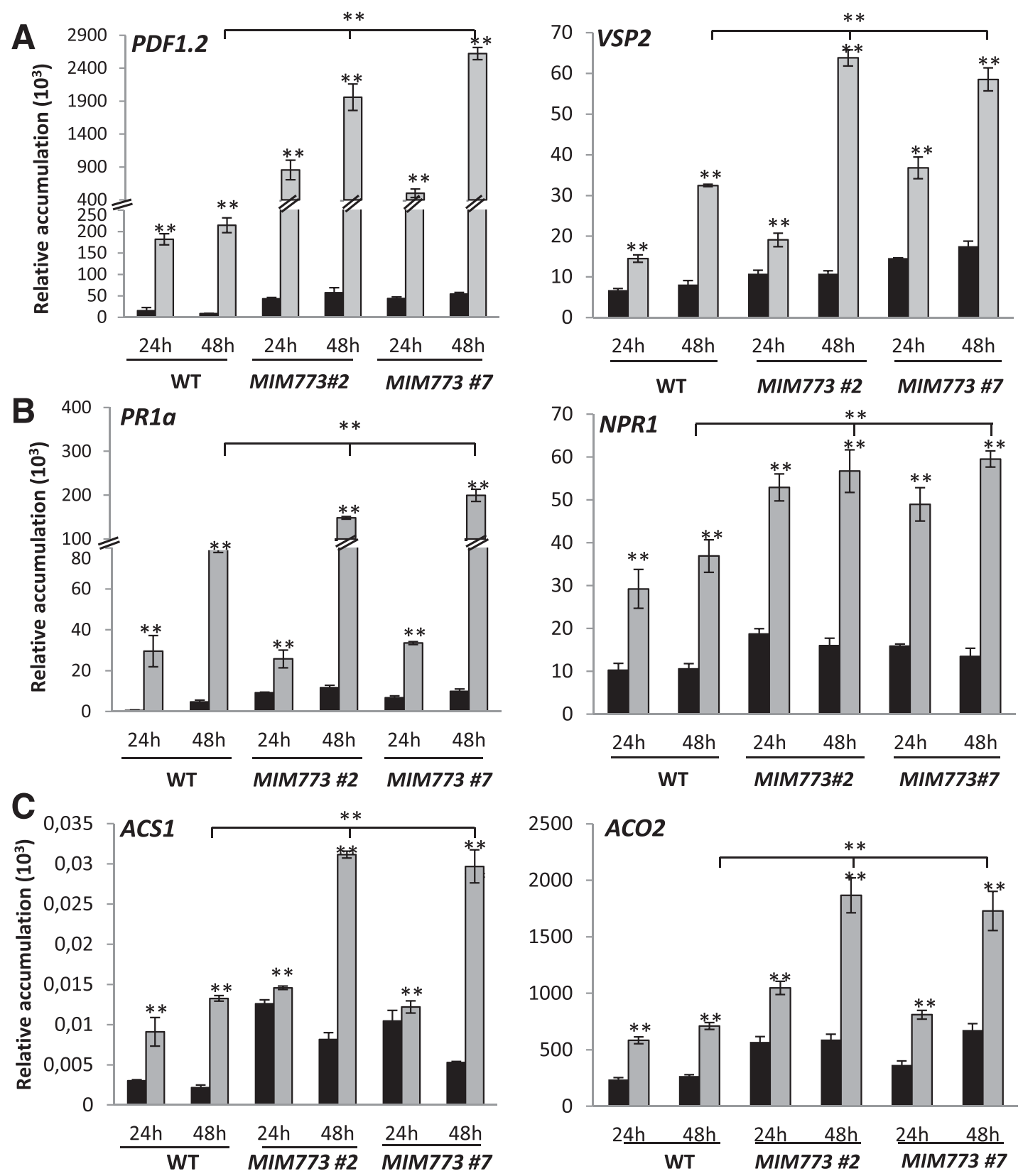

Fig. 7. Accumulation of defense marker genes in wild-type and $M I M 773$ plants. A and $\mathbf{B}$, Reverse transcription-quantitative polymerase chain reaction analysis showing the accumulation of transcripts for genes that are regulated by the jasmonic acid and ethylene (JA/ET)-dependent (PDF1.2), JA-dependent (VSP2), and salicylic acid-dependent (PRIa, NPRI) signaling pathways at the indicated timepoints after inoculation with Plectosphaerrella cucumerina spores. C, Analysis of the ACS1 and ACO2 genes involved in ET biosynthesis is shown. Mock- and P.cucumerina-inoculated plants are represented by black and gray bars, respectively. Results from two of the three MIM773 lines analyzed are shown (similar results were obtained for the third line). Histograms show the mean \pm standard deviation of three biological replicates, each with 12 plants per genotype. Statistical significance was determined by analysis of variance (asterisks [**] indicate $P \leq 0.01$, P. cucumerina- vs. mock-inoculated; wild-type $P$. cucumerina-inoculated $48 \mathrm{~h}$ vs. MIM773 P. cucumerina-inoculated 48 h). 
overall status of DNA methylation (Deleris et al. 2016). Typically, DNA methylation represses gene expression, whereas hypo-methylation is frequently associated with transcriptional upregulation. The dynamic regulation and functional consequences of DNA methylation are, however, substantially more complex. For instance, in flowering plants there are at least five distinct classes of methylated genes, including gene body methylated $(\mathrm{gbM})$ genes in which there is an enrichment of CG DNA methylation within the transcribed regions and depletion at the transcriptional start and termination sites (Bewick and Schmitz 2017). Although the function of gbM remains elusive, it was proposed that gbM serves to regulate splicing and gene expression (Regulski et al. 2013; Zilberman et al. 2008). To note, infection of Arabidopsis plants with $P$. syringae results in dynamic changes in DNA methylation, which in turn lead to the transcriptional activation of defense-related genes (Dowen et al. 2012; Pavet et al. 2006; Yu et al. 2013). Similarly, about half of all genes that respond to infection by the oomycete pathogen Hyaloperonospora arabidopsidis are also altered in mutants with DNA methylation and gene silencing defects (López Sánchez et al. 2016). Such mutants are also affected in pathogen resistance, but they can be either more resistant or more susceptible, depending on the pathogen lifestyle. The hyper-methylated ros1 (REPRESSOR OF SILENCING 1) mutant (affected in DNA demethylation) has increased susceptibility to the biotrophic oomycete $H$. arabidopsidis but enhanced resistance to the necrotrophic fungus $P$. cucumerina (López-Sánchez et al. 2016). Conversely, the hypo-methylated nrpel RdDM (defective in RNA-directed DNA methylation) mutant exhibited enhanced susceptibility to $P$. cucumerina infection but resistance to $H$. arabidopsidis (López Sánchez et al. 2016).

Consistent with results reported by López Sánchez et al. (2016), upregulation of MET2 through interference with miR773 activity confers resistance to $P$. cucumerina, whereas downregulation of MET2 expression through MIR773 overexpression increases susceptibility to infection. These observations reinforce the interpretation that DNA methylation controls basal resistance against $P$. cucumerina in Arabidopsis. DNA methylation may exert persistent control over defense genes in the absence of infection, with infection triggering both DNA methylation and gene-expression changes. DNA methylation might modulate immune responses, either by modifying the activity of gene promoters or bodies (or both) or by regulating the transcriptional status of transposable elements at specific loci. For instance, the methylation status of the COPIA retrotransposon has been shown to control the expression of RPP7 (RECOGNITION OF PERONOSPORA PARASITICA 7), a disease resistance gene encoding an immune receptor conferring resistance to $H$. arabidopsidis in Arabidopsis (Tsuchiya and Eulgem 2013).

To conclude, our results lend further support to findings that miR773a is an important component in the regulation of PTI responses. Given the absence of obvious defects in noninfected plants with altered miR773 or miR773 target activity, manipulation of miR773 or its targets may provide a new strategy for engineering crop protection.

\section{MATERIALS AND METHODS}

\section{Plant material and growth conditions.}

Arabidopsis (Arabidopsis thaliana) Col-0 plants were grown on a mixture of soil, perlite, and vermiculite $(2: 1: 1)$ under neutral day conditions ( $12 \mathrm{~h}$ of light and $12 \mathrm{~h}$ of dark), at $22^{\circ} \mathrm{C}$ day and $20^{\circ} \mathrm{C}$ night temperatures, and $60 \%$ relative humidity. The production of MIM lines (Col-0 background) was previously described (Todesco et al. 2010). Rosette diameter and leaf number were determined in 3-week-old plants, using Image J. The Arabidopsis mutants used in this work, met2-1 and met2-2 (SALK_010893 and SALK_010896, respectively) were grown as described above. Primers used for genotyping the T-DNA insertion mutants are indicated in Supplementary Table S2.

For MIR773A overexpression, a 364-bp genomic sequence containing the miR773a precursor was obtained by PCR from Arabidopsis genomic DNA, using gene-specific primers. The miR773a precursor sequence was cloned into the pCAMBIA1300 vector under the control of the $35 \mathrm{~S} \mathrm{CaMV}$ promoter. The floral dip method was used for Arabidopsis (Col-0) transformation. As a control, Arabidopsis plants were transformed with the pCAMBIA1300 empty vector.

\section{Infection assays and elicitor treatment.}

Fungi were grown at $28^{\circ} \mathrm{C}$ on PDA (potato dextrose agar) supplemented with chloramphenicol. Spores were collected by adding sterile water to the surface of the mycelium, adjusting to the appropriate concentration using a Bürker counting chamber. For infection experiments, control (wild-type, empty vector) and MIM plants (homozygous lines) were grown for 3 weeks and were then spray-inoculated with a spore suspension of $P$. cucumerina at the appropriate concentration or were mockinoculated. Progression of the infection was examined at different days postinoculation. In the case of $F$. oxysporum f. sp. conglutinans, the spore suspension was applied to the soil near the roots $\left(200 \mu \mathrm{l}, 1 \times 10^{6}\right.$ spores per milliliter). Infections with C. higginsianum were performed by drop-inoculation of the leaves $\left(10 \mu \mathrm{l}, 5 \times 10^{5}\right.$ spores per milliliter). Fungal- and mockinoculated plants were allowed to continue growth under high humidity. Statistically significant differences among the inoculated Arabidopsis genotypes were determined by one-way analysis of variance (ANOVA). Disease severity and lesion area were determined using digital imaging software (Assess 2.0, American Phytopathological Society). The relative quantification of fungal DNA in infected leaves was carried out by qPCR as described (Sánchez-Vallet et al. 2010; Soto-Suárez et al. 2017). For elicitor treatment, Arabidopsis plants were sprayed with an elicitor suspension obtained from $P$. cucumerina $(300 \mu \mathrm{g} / \mathrm{ml})$ as previously described (Coca and San Segundo 2010).

\section{Plant tissue staining.}

Trypan blue staining was performed as previously described (Ramírez et al. 2010). Stained material was observed using a Zeiss Axiophot microscope under bright-field illumination. At least 15 plants per genotype were analyzed.

Callose deposition was determined by aniline blue staining. For this, chlorophyll was removed, with $70 \%$ ethanol, from leaves that were then incubated in $70 \mathrm{mM}$ phosphate buffer ( $\mathrm{pH} 9.0)$ supplemented with aniline blue $(0.01 \% \mathrm{wt} / \mathrm{vol})$ with vacuum for $30 \mathrm{~min}$. Samples were maintained in dark conditions for $2 \mathrm{~h}$. Leaves were observed with an epifluorescence microscope (Zeiss Axiophot microscope) under UV illumination. Callose deposition was quantified by determining the relative number of callose-corresponding pixels relative to the total number of pixels covering the plant material on digital photographs (Luna et al. 2011).

DAB (3,3'-diaminobenzidine) staining was used to determine $\mathrm{H}_{2} \mathrm{O}_{2}$ levels. For this, Arabidopsis plants were immersed in a DAB solution $(1 \mathrm{mg} / \mathrm{ml})$ with vacuum for $30 \mathrm{~min}$, were maintained in the dark overnight, and were washed with $70 \%$ ethanol for $1 \mathrm{~h}$. Plants were observed using an Olympus DP71 microscope under bright-field illumination. DAB staining intensities were quantified from digital photographs by the number of dark-brown DAB pixels relative to the leaf surface. 
Callose measurements and $\mathrm{H}_{2} \mathrm{O}_{2}$ quantifications were made on at least 15 different seedlings per genotype. Statistically significant differences were determined by one-way ANOVA test.

\section{Gene expression analyses.}

Total RNA was extracted using TRIzol reagent (Invitrogen). First-strand cDNA was synthesized from TURBO DNAse (Ambion) treated total RNA $(1 \mu \mathrm{g})$ with High Capacity cDNA reverse transcription kit (Applied Biosystems) and oligo-dT. Reverse transcription (RT)-qPCR tests were performed in optical 96-well plates, in a Light Cycler 480, using SYBR green (Roche). Primers were designed using Oligo Analyzer software (Integrated DNA Technologies). The $\beta$-tubulin2 (At5g05620) expression was used to normalize the transcript level in each sample. Three independent biological replicates with three technical replicates were analyzed. An ANOVA test was used to evaluate differences in gene expression. Accumulation of mature miR773 sequences was determined by stem-loop RT-qPCR (Varkonyi-Gasic et al. 2007). Nucleotide sequencing confirmed the specific amplification of miR773 sequences.

For Northern blot analysis, the small RNA fraction obtained from total RNA $(600 \mu \mathrm{g})$ was fractioned in a $17.5 \%$ polyacrylamide gel containing $8 \mathrm{M}$ urea and was transferred to nylon membranes. Probes were 3'-labeled with digoxigenin (DIG), using the DIG oligonucleotide 3 '-end labeling kit, following manufacturer's instructions (Roche).

\section{MiR773 in situ hybridization.}

Plants grown in in vitro conditions for 15 days were inoculated with $P$. cucumerina spores or were mock-inoculated. Mature leaves were harvested at $24 \mathrm{~h}$ postinoculation and were analyzed separately for in situ miR773 detection. Fixation and sample processing was performed as described by Wang et al. (2008). Hybridization was carried out with a $3^{\prime}$ DIG-labeled locked nucleic acid miR773a probe (Exiqon) overnight at $37^{\circ} \mathrm{C}$. Detection of the hybridized probe was performed using an antibody solution (anti-DIG, alkaline phosphatase conjugated antibody) and the color substrate NBT (4-nitroblue-tetrazolium chloride).

\section{ACKNOWLEDGMENTS}

We thank A. Molina (Centro de Biotecnologia y Genomica de Plantas, Madrid) for the P. cucumerina strain and the agb1.2 mutant, A. di Pietro (Universidad de Cordoba) for F. oxysporum f. sp. conglutinans, R. J. O'Connell (Max Planck Institute for Plant Breeding Research, Koln, Germany) for Colletotrichum higginsianum, and members of the MoRE Lab (I. Rubio-Somoza) for critically reading the manuscript. R. Salvador-Guirao is a recipient of a Ph.D. grant from the Ministerio de Economía y Competitividad, Formacion de Personal Investigador (BES-2013-065521). This work was supported by the Spanish Ministry of Economy and Competitiveness (MINECO) and the European Regional Development's funds (FEDER) (BIO2012-32838 and BIO2015-67212-R to B. San Segundo; BFU2014-58361-JIN to I. RubioSomoza), and the Deutsche Forschungsgemeinschaft (grant SFB1101) and European Commission to D. Weigel (advanced grant IMMUNEMESIS). We thank the Max Planck Society and the Prince of Asturias Foundation for a MaxPlanck-Prince of Asturias Award Mobility Grant to R. Salvador-Guirao. We acknowledge financial support from MINECO through the "Severo Ochoa Programme for Centres of Excellence in R\&D 2016-2019 (SEV-2015-0533)" and the CERCA Programme from the Generalitat de Catalunya.

\section{LITERATURE CITED}

Axtell, M. J. 2013. Classification and comparison of small RNAs from plants. Annu. Rev. Plant Biol. 64:137-159.

Baldrich, P., Kakar, K., Siré, C., Moreno, A. B., Berger, A., García-Chapa, M., López-Moya, J. J., Riechmann, J. L., and San Segundo, B. 2014. Small RNA profiling reveals regulation of Arabidopsis miR168 and heterochromatic siRNA415 in response to fungal elicitors. BMC Genomics 15:1083.
Bewick, A. J., and Schmitz, R. J. 2017. Gene body DNA methylation in plants. Curr. Opin. Plant Biol. 36:103-110.

Bigeard, J., Colcombet, J., and Hirt, H. 2015. Signaling mechanisms in pattern-triggered immunity (PTI). Mol. Plant 8:521-539.

Boccara, M., Sarazin, A., Thiébeauld, O., Jay, F., Voinnet, O., Navarro, L., and Colot, V. 2014. The Arabidopsis miR472-RDR6 silencing pathway modulates PAMP- and effector-triggered immunity through the posttranscriptional control of disease resistance genes. PLoS Pathog. 10: e1003883.

Boller, T., and He, S. Y. 2009. Innate immunity in plants: An arms race between pattern recognition receptors in plants and effectors in microbial pathogens. Science 324:742-744.

Bravo Ruiz, G., Di Pietro, A., and Roncero, M. I. G. 2016. Combined action of the major secreted exo- and endopolygalacturonases is required for full virulence of Fusarium oxysporum. Mol. Plant Pathol. 17:339-353.

Brodersen, P., Sakvarelidze-Achard, L., Bruun-Rasmussen, M., Dunoyer, P., Yamamoto, Y. Y., Sieburth, L., and Voinnet, O. 2008. Widespread translational inhibition by plant miRNAs and siRNAs. Science 320 : 1185-1190.

Campo, S., Peris-Peris, C., Siré, C., Moreno, A. B., Donaire, L., Zytnicki, M., Notredame, C., Llave, C., and San Segundo, B. 2013. Identification of a novel microRNA (miRNA) from rice that targets an alternatively spliced transcript of the Nramp6 (Natural resistance-associated macrophage protein 6) gene involved in pathogen resistance. New Phytol. 199: 212-227.

Chiou, T.-J., Aung, K., Lin, S.-I., Wu, C.-C., Chiang, S.-F., and Su, C.-L. 2006. Regulation of phosphate homeostasis by microRNA in Arabidopsis. Plant Cell 18:412-421.

Coca, M., and San Segundo, B. 2010. AtCPK1 calcium-dependent protein kinase mediates pathogen resistance in Arabidopsis. Plant J. 63: 526-540.

Cui, H., Tsuda, K., and Parker, J. E. 2015. Effector-triggered immunity: From pathogen perception to robust defense. Annu. Rev. Plant Biol. 66:487-511.

De Vleesschauwer, D., Xu, J., and Höfte, M. 2014. Making sense of hormone-mediated defense networking: From rice to Arabidopsis. Front Plant Sci. 5:611.

Deleris, A., Halter, T., and Navarro, L. 2016. DNA methylation and demethylation in plant immunity. Annu. Rev. Phytopathol. 54:579-603.

Denancé, N., Sánchez-Vallet, A., Goffner, D., and Molina, A. 2013. Disease resistance or growth: The role of plant hormones in balancing immune responses and fitness costs. Front. Plant Sci. 4:1-12.

Dowen, R. H., Pelizzola, M., Schmitz, R. J., Lister, R., Dowen, J. M., Nery, J. R., Dixon, J. E., and Ecker, J. R. 2012. Widespread dynamic DNA methylation in response to biotic stress. Proc. Natl. Acad. Sci. U.S.A 109:E2183-E2191.

Fahlgren, N., Howell, M. D., Kasschau, K. D., Chapman, E. J., Sullivan, C. M., Cumbie, J. S., Givan, S. A., Law, T. F., Grant, S. R., Dangl, J. L., and Carrington, J. C. 2007. High-throughput sequencing of Arabidopsis microRNAs: Evidence for frequent birth and death of MIRNA genes. PLoS One 2:e219.

García-Andrade, J., Ramírez, V., Flors, V., and Vera, P. 2011. Arabidopsis ocp3 mutant reveals a mechanism linking ABA and JA to pathogeninduced callose deposition. Plant J. 67:783-794.

Glazebrook, J. 2005. Contrasting mechanisms of defense against biotrophic and necrotrophic pathogens. Annu. Rev. Phytopathol. 43:205-227.

Huang, J., Yang, M., and Zhang, X. 2016. The function of small RNAs in plant biotic stress response. J. Integr. Plant Biol. 58:312-327.

Jagadeeswaran, G., Saini, A., and Sunkar, R. 2009. Biotic and abiotic stress down-regulate miR398 expression in Arabidopsis. Planta 229:10091014

Jones, J. D. G., and Dangl, J. L. 2006. The plant immune system. Nature 444:323-329

Katiyar-Agarwal, S., and Jin, H. 2010. Role of small RNAs in host-microbe interactions. Annu. Rev. Phytopathol. 48:225-246.

Lee, H. J., Park, Y. J., Kwak, K. J., Kim, D., Park, J. H., Lim, J. Y., Shin, C., Yang, K.-Y., and Kang, H. 2015. MicroRNA844-guided downregulation of cytidinephosphate diacylglycerol synthase3 (CDS3) mRNA affects the response of Arabidopsis thaliana to bacteria and fungi. Mol. PlantMicrobe Interact. 28:892-900.

Li, C., and Zhang, B. 2016. MicroRNAs in control of plant development. J. Cell. Physiol. 231:303-313.

Li, S., Liu, L., Zhuang, X., Yu, Y., Liu, X., Cui, X., Ji, L., Pan, Z., Cao, X., Mo, B., Zhang, F., Raikhel, N., Jiang, L., and Chen, X. 2013. MicroRNAs inhibit the translation of target mRNAs on the endoplasmic reticulum in Arabidopsis. Cell 153:562-574.

Li, Y., Lu, Y. G., Shi, Y., Wu, L., Xu, Y. J., Huang, F., Guo, X. Y., Zhang, Y., Fan, J., Zhao, J. Q., Zhang, H. Y., Xu, P. Z., Zhou, J. M., Wu, X. J., Wang, P. R., and Wang, W. M. 2014. Multiple rice microRNAs are involved in 
immunity against the blast fungus Magnaporthe oryzae. Plant Physiol. 164:1077-1092.

Li, Y., Zhang, Q., Zhang, J., Wu, L., Qi, Y., and Zhou, J.-M. 2010. Identification of microRNAs involved in pathogen-associated molecular pattern-triggered plant innate immunity. Plant Physiol. 152:2222-2231.

Llave, C., Xie, Z., Kasschau, K. D., and Carrington, J. C. 2002. Cleavage of Scarecrow-like mRNA targets directed by a class of Arabidopsis miRNA. Science 297:2053-2056.

Llorente, F., Alonso-Blanco, C., Sánchez-Rodriguez, C., Jordá, L., and Molina, A. 2005. ERECTA receptor-like kinase and heterotrimeric G protein from Arabidopsis are required for resistance to the necrotrophic fungus Plectosphaerella cucumerina. Plant J. 43:165-180.

Llorente, F., Muskett, P., Sánchez-Vallet, A., López, G., Ramos, B., SánchezRodríguez, C., Jordá, L., Parker, J., and Molina, A. 2008. Repression of the auxin response pathway increases Arabidopsis susceptibility to necrotrophic fungi. Mol. Plant 1:496-509.

López Sánchez, A., Stassen, J. H., Furci, L., Smith, L. M., and Ton, J. 2016. The role of DNA (de)methylation in immune responsiveness of Arabidopsis. Plant J. 88:361-374.

Luna, E., Pastor, V., Robert, J., Flors, V., Mauch-Mani, B., and Ton, J. 2011. Callose deposition: A multifaceted plant defense response. Mol. PlantMicrobe Interact. 24:183-193.

Mauch-Mani, B., and Slusarenko, A. 1994. Systemic acquired resistance in Arabidopsis thaliana induced by a predisposing infection with a pathogenic isolate of Fusarium oxysporum. Mol. Plant-Microbe Interact. 7:378-383.

Morales, J., Kadota, Y., Zipfel, C., Molina, A., and Torres, M. 2016. The Arabidopsis NADPH oxidases $R b o h D$ and $R b o h F$ display differential expression patterns and contributions during plant immunity. J. Exp. Bot. 67:1-14.

Navarro, L., Dunoyer, P., Jay, F., Arnold, B., Dharmasiri, N., Estelle, M., Voinnet, O., and Jones, J. D. G. 2006. A plant miRNA contributes to antibacterial resistance by repressing auxin signaling. Science 312:436-439.

Niu, D., Lii, Y. E., Chellappan, P., Lei, L., Peralta, K., Jiang, C., Guo, J., Coaker, G., and Jin, H. 2016. miRNA863-3p sequentially targets negative immune regulator ARLPKs and positive regulator SERRATE upon bacterial infection. Nat. Commun. 7:11324.

O’Connell, R., Herbert, C., Sreenivasaprasad, S., Khatib, M., EsquerréTugayé, M. T., and Dumas, B. 2004. A novel Arabidopsis-Colletotrichum pathosystem for the molecular dissection of plant-fungal interactions. Mol. Plant-Microbe Interact. 17:272-282.

Palm, M. E. 1995. Plectosporium, a new genus for Fusarium tabacinum, the anamorph of Plectosphaerella cucumerina. Mycologia 87:397-406.

Park, Y. J., Lee, H. J., Kwak, K. J., Lee, K., Hong, S. W., and Kang, H. 2014. MicroRNA400-guided cleavage of pentatricopeptide repeat protein mRNAs renders Arabidopsis thaliana more susceptible to pathogenic bacteria and fungi. Plant Cell Physiol. 55:1660-1668.

Pastor, V., Luna, E., Ton, J., Cerezo, M., García-Agustín, P., and Flors, V. 2013. Fine tuning of reactive oxygen species homeostasis regulates primed immune responses in Arabidopsis. Mol. Plant-Microbe Interact. 26:1334-1344.

Pavet, V., Quintero, C., Cecchini, N. M., Rosa, A. L., and Alvarez, M. E. 2006. Arabidopsis displays centromeric DNA hypomethylation and cytological alterations of heterochromatin upon attack by Pseudomonas syringae. Mol. Plant-Microbe Interact. 19:577-587.

Pétriacq, P., Stassen, J. H., and Ton, J. 2016. Spore density determines infection strategy by the plant pathogenic fungus Plectosphaerella cucumerina. Plant Physiol. 170:2325-2339.

Pumplin, N., and Voinnet, O. 2013. RNA silencing suppression by plant pathogens: Defence, counter-defence and counter-counter-defence. Nat. Rev. Microbiol. 11:745-760.

Ramírez, V., Van der Ent, S., García-Andrade, J., Coego, A., Pieterse, C. M. J., and Vera, P. 2010. OCP3 is an important modulator of NPR1mediated jasmonic acid-dependent induced defenses in Arabidopsis. BMC Plant Biol. 10:199.

Regulski, M., Lu, Z., Kendall, J., Donoghue, M. T. A., Reinders, J., Llaca, V., Deschamps, S., Smith, A., Levy, D., McCombie, W. R., Tingey, S., Rafalski, A., Hicks, J., Ware, D., and Martienssen, R. A. 2013. The maize methylome influences mRNA splice sites and reveals widespread paramutation-like switches guided by small RNA. Genome Res. 23:1651-1662.

Robert-Seilaniantz, A., Grant, M., and Jones, J. D. G. 2011. Hormone crosstalk in plant disease and defense: More than just jasmonate-salicylate antagonism. Annu. Rev. Phytopathol. 49:317-343.

Rogers, K., and Chen, X. 2013. Biogenesis, turnover, and mode of action of plant microRNAs. Plant Cell 25:2383-2399.

Rubio-Somoza, I., and Weigel, D. 2011. MicroRNA networks and developmental plasticity in plants. Trends Plant Sci. 16:258-264.

Sánchez-Vallet, A., López, G., Ramos, B., Delgado-Cerezo, M., Riviere, M. P., Llorente, F., Fernández, P. V., Miedes, E., Estevez, J. M., Grant, M., and Molina, A. 2012. Disruption of abscisic acid signaling constitutively activates Arabidopsis resistance to the necrotrophic fungus Plectosphaerella cucumerina. Plant Physiol. 160:2109-2124.

Sánchez-Vallet, A., Ramos, B., Bednarek, P., López, G., PiślewskaBednarek, M., Schulze-Lefert, P., and Molina, A. 2010. Tryptophanderived secondary metabolites in Arabidopsis thaliana confer non-host resistance to necrotrophic Plectosphaerella cucumerina fungi. Plant J. 63:115-127.

Seo, J. K., Wu, J., Lii, Y., Li, Y., and Jin, H. 2013. Contribution of small RNA pathway components in plant immunity. Mol. Plant-Microbe Interact. 26:617-625.

Shivaprasad, P. V., Chen, H. M., Patel, K., Bond, D. M., Santos, B. A. C. M., and Baulcombe, D. C. 2012. A microRNA superfamily regulates nucleotide binding site-leucine-rich repeats and other mRNAs. Plant Cell 24:859-874.

Soto-Suárez, M., Baldrich, P., Weigel, D., Rubio-Somoza, I., and San Segundo, B. 2017. The Arabidopsis miR396 mediates pathogenassociated molecular pattern-triggered immune responses against fungal pathogens. Sci. Rep. 7:44898.

Staiger, D., Korneli, C., Lummer, M., and Navarro, L. 2013. Emerging role for RNA-based regulation in plant immunity. New Phytol. 197:394-404.

Sunkar, R., Li, Y. F., and Jagadeeswaran, G. 2012. Functions of microRNAs in plant stress responses. Trends Plant Sci. 17:196-203.

Todesco, M., Rubio-Somoza, I., Paz-Ares, J., and Weigel, D. 2010. A collection of target mimics for comprehensive analysis of microRNA function in Arabidopsis thaliana. PLoS Genet. 6:e1001031.

Ton, J., and Mauch-Mani, B. 2004. $\beta$-amino-butyric acid-induced resistance against necrotrophic pathogens is based on ABA-dependent priming for callose. Plant J. 38:119-130.

Torres, M. A., Jones, J. D. G., and Dangl, J. L. 2006. Reactive oxygen species signaling in response to pathogens. Plant Physiol. 141:373-378.

Tsuchiya, T., and Eulgem, T. 2013. An alternative polyadenylation mechanism coopted to the Arabidopsis RPP7 gene through intronic retrotransposon domestication. Proc. Natl. Acad. Sci. U.S.A. 110: E3535-E3543.

van Loon, L. C., Rep, M., and Pieterse, C. M. J. 2006. Significance of inducible defense-related proteins in infected plants. Annu. Rev. Phytopathol. 44:135-162.

Varkonyi-Gasic, E., Wu, R., Wood, M., Walton, E. F., and Hellens, R. P. 2007. Protocol: A highly sensitive RT-PCR method for detection and quantification of microRNAs. Plant Methods 3:12.

Wang, J., Schwab, R., Czech, B., Mica, E., and Weigel, D. 2008. Dual effects of miR156-targeted SPL genes and CYP78A5/KLUH on plastochron length and organ size in Arabidopsis thaliana. Plant Cell 20: 1231-1243.

Yu, A., Lepère, G., Jay, F., Wang, J., Bapaume, L., Wang, Y., Abraham, A. L., Penterman, J., Fischer, R. L., Voinnet, O., and Navarro, L. 2013. Dynamics and biological relevance of DNA demethylation in Arabidopsis antibacterial defense. Proc. Natl. Acad. Sci. U.S.A. 110:23892394.

Zilberman, D., Coleman-Derr, D., Ballinger, T., and Henikoff, S. 2008. Histone H2A.Z and DNA methylation are mutually antagonistic chromatin marks. Nature 456:125-129.

Zipfel, C., Robatzek, S., Navarro, L., Oakeley, E. J., Jones, J. D. G., Felix, G., and Boller, T. 2004. Bacterial disease resistance in Arabidopsis through flagellin perception. Nature 428:764-767. 